\title{
Guanidinoacetic Acid Consumption via Regular Diet in Adults
}

\author{
Sergej M. Ostojic ${ }^{a, b}, c$ Valdemar Stajer ${ }^{b}$ Laszlo Ratgeber ${ }^{c}$ Jozsef Betlehem ${ }^{c}$ \\ Pongrac Acs ${ }^{\mathrm{C}}$ \\ aDepartment of Nutrition and Public Health, University of Agder, Kristiansand, Norway; ${ }^{\mathrm{b}}$ Faculty of Sport and \\ Physical Education, University of Novi Sad, Novi Sad, Serbia; 'Faculty of Health Sciences, University of Pecs, Pecs, \\ Hungary
}

\section{Dear Editor,}

Guanidinoacetic acid (GAA; also known as glycocyamine) is a natural amino acid-like derivative of glycine and $\mathrm{L}$-arginine. GAA plays several metabolic roles in the human body [1], with creatine synthesis being recognized as a crucial pathway of GAA utilization. A daily output of GAA largely depends on endogenous synthesis that occurs mainly in the kidney and pancreas [2], while diet has traditionally been considered as an irrelevant source of GAA in humans. However, several common foods can contain GAA (see: https://foodb.ca/compounds/ FDB005417), suggesting that exogenous supplies may contribute to a total GAA out-turn; no studies so far provided data about dietary GAA exposure in the general public. Given a fundamental role of creatine in human nutrition and energy metabolism [3], accounting for food-driven GAA might add up to a well-balanced creatine homeostatic load. In this cross-sectional study, we aimed to quantify the amount of GAA consumed through the regular diet in the US adult population, using opensource data from the 2017-2018 National Health and Nutrition Examination Survey (NHANES).

The NHANES is a continuous survey program directed by the US National Center for Health Statistics to evaluate the health and nutritional status of the non-institutionalized civilian resident population of the USA in 2-year cycles, from 1971 onwards. The NHANES 2017-2018 round selected 16,211 persons aged 0-80+ years from 30 different survey locations. The approval to conduct NHANES 2017-2018 was granted by the National Center for Health Statistics Research Ethics Review Board (Protocol \#201801, and Continuation of Protocol \#2011-17). Of individuals selected, we extracted data for 4,772 adults who completed a 24-h Dietary Recall, a structured interview intended to collect detailed information about all foods and beverages consumed by the respondent in the past $24 \mathrm{~h}$. Initially, GAA-containing foods (e.g., meat, poultry, fish, and mixtures; milk and milk products; apple and loquat) were distinguished using unique 8-digit food code numbers from the USA Department of Agriculture proxy-interview entries for individual foods. Afterwards, the gram weight of each GAA-containing food was noted down, and total daily intake of those foods were computed for each respondent by merging all relevant food items. Individual values for GAA consumed per day were calculated using the average amount of GAA for apple $(1.2 \mu \mathrm{g} / \mathrm{kg})$ [4], diary group $(0.3 \mathrm{mg} / \mathrm{kg})$ [5], and meat-based foods $(42.9 \mathrm{mg} / \mathrm{kg})$ [6] across all GAA-containing food sources.

GAA-containing foods comprise $22.6 \%$ of all foods consumed, with the most prevalent dietary source of GAA were meat-based foods (51.6\%), followed by a diary group (42.6\%), and apple (5.9\%). The mean dietary intake of GAA was $9.9 \pm 9.7 \mathrm{mg}$ per day (95\% CI, from 9.6 to 10.1 ), with the highest consumption recorded in a 50 -year karger@karger.com

(c) 2021 S. Karger AG, Basel

www.karger.com/anm

Karger"
Correspondence to:

Sergej M. Ostojic, sergej.ostojic@uia.no 
old man (116.0 mg/day). Men consumed more GAA than women $(11.9 \pm 10.9 \mathrm{~g} /$ day vs. $8.0 \pm 7.9 \mathrm{~g} /$ day; $p<0.001)$, and a significant age-associated reduction in dietary GAA intake was found $(r=-0.06, p<0.01)$.

We found here that the dietary exposure to GAA is relatively low among the US adults, with the average intake ( $\sim 10 \mathrm{mg} /$ day) only contributes marginally to the total daily turnover of creatine. Since the amount of creatine that needs to be supplied by a diet and synthesis is $\sim 2$ g per day for an average adult [2], food-driven GAA appears to provide approximately $0.5 \%$ of creatine homeostatic load through a regular diet. A minimal amount of GAA provided via food sources implies that de novo synthesis of GAA from glycine and L-arginine must provide a majority of this compound; however, no data are currently available concerning the daily requirements and replacement of GAA. Besides, the food composition tables contain very limited information about the GAA content of foods and beverages which may underestimate total dietary intakes of GAA. Interestingly, a mean intake in women is about $67 \%$ of intake in men, which may illustrate a gender-specific metabolic demand on GAA. Further understanding of the importance of GAA supplied via diet remains of high importance, having in mind that supplying GAA as a food supplement might be a superior vehicle to support creatine output [7].

\section{Statement of Ethics}

The study was conducted according to the guidelines of the Declaration of Helsinki. The ethical approval to conduct the NHANES rounds was granted by the US National Center for Health Statistics Research Ethics Review Board (Protocol \#201801, and Continuation of Protocol \#2011-17), and written informed consent was obtained from all respondents.

\section{Conflict of Interest Statement}

S.M.O. serves as a member of the Scientific Advisory Board on creatine in health and medicine (AlzChem LLC). S.M.O. owns patent "Sports Supplements Based on Liquid Creatine" at European Patent Office (WO2019150323 A1), and active patent application "Synergistic Creatine" at UK Intellectual Property Office (GB2012773.4). S.M.O. has served as a speaker at Abbott Nutrition, a consultant of Allied Beverages Adriatic and IMLEK, and an advisory board member for the University of Novi Sad School of Medicine, and has received research funding related to creatine from the Serbian Ministry of Education, Science, and Technological Development, Provincial Secretariat for Higher Education and Scientific Research, AlzChem GmbH, KW Pfannenschmidt $\mathrm{GmbH}$, Monster Beverage Corporation, and ThermoLife International LLC. S.M.O. does not own stocks and shares in any organization. V.S., L.R., J.B., and P.A. declare no conflict of interest.

\section{Funding Sources}

This work was not funded by any agency in the public, commercial, or not-for-profit sectors.

\section{Author Contributions}

The authors' responsibilities were as follows: S.M.O. designed and wrote the draft of the manuscript and has primary responsibility for the final content; and all authors read and approved the final version of the manuscript.

\section{Data Availability Statement}

Data described in the manuscript will be made available upon request.

\section{References}

1 Ostojic SM. Advanced physiological roles of guanidinoacetic acid. Eur J Nutr. 2015;54(8): 1211-5.

2 Brosnan JT, da Silva RP, Brosnan ME. The metabolic burden of creatine synthesis. Amino Acids. 2011;40(5):1325-31.

$3 \mathrm{Wu}$ G. Important roles of dietary taurine, creatine, carnosine, anserine and 4-hydroxyproline in human nutrition and health. Amino Acids. 2020;52(3):329-60.
4 Kato T, Yamagata M, Tsukahara S. Guanidine compounds in fruit trees and their seasonal variations in citrus (citrus unshiu marc.) Engei Gakkai Zasshi. 1986;55(2):169-73.

5 Edison EE, Brosnan ME, Aziz K, Brosnan JT. Creatine and guanidinoacetate content of human milk and infant formulas: implications for creatine deficiency syndromes and amino acid metabolism. Br J Nutr. 2013;110(6): 1075-8.
6 European Food Safety Authority. Panel on additives and products or substances used in animal feed (FEEDAP). Safety and efficacy of guanidinoacetic acid for chickens for fattening, breeder hens and roosters, and pigs. EFSA J. 2016;14(2):4394.

7 Semeredi S, Stajer V, Ostojic J, Vranes M, Ostojic SM. Guanidinoacetic acid with creatine compared with creatine alone for tissue creatine content, hyperhomocysteinemia, and exercise performance: a randomized, doubleblind superiority trial. Nutrition. 2019 Jan;57: 162-6. 